\title{
Comparison of Two Predictive Models of Sepsis in Critically III Patients Based on the Combined Use of Inflammatory Markers
}

\author{
Xiaoming $\mathrm{Li}^{1,2}$, Chao Liu $\mathbb{D}^{2}$, Xiaoli Wang ${ }^{1}$, Zhi Mao², Hongyu $\mathrm{Yi}^{1}$, Feihu Zhou $\mathbb{D}^{2}$ \\ 'Medical School of Chinese PLA, Beijing, People's Republic of China; ${ }^{2}$ Department of Critical Care Medicine, The First Medical Centre, Chinese PLA \\ General Hospital, Beijing, People's Republic of China \\ Correspondence: Feihu Zhou, Critical Care Medicine, The First Medical Centre, Chinese People's Liberation Army General Hospital, 28 Fu-Xing \\ Road, Beijing, 100853, People's Republic of China, Tel +86-I0-66938I48, Fax +86-I0-882I9862, Email feihuzhou30I@I26.com
}

\begin{abstract}
Background: Sepsis is a systemic inflammatory response due to infection, resulting in organ dysfunction. Timely targeted interventions can improve prognosis. Inflammation plays a crucial role in the process of sepsis. To identify potential sepsis early, we developed and validated a nomogram model and a simple risk scoring model for predicting sepsis in critically ill patients.

Methods: The medical records of adult patients admitted to our intensive care unit (ICU) from August 2017 to December 2020 were analyzed. Patients were randomly divided into a training cohort (70\%) and a validation cohort (30\%). A nomogram model was developed through multivariate logistic regression analysis. The continuous variables included in nomogram model were transformed into dichotomous variables. Then, a multivariable logistic regression analysis was performed based on these dichotomous variables, and the odds ratio $(\mathrm{OR})$ for each variable was used to construct a simple risk scoring model. The receiver operating characteristic curves (ROC) were constructed, and the area under the curve (AUC) was calculated.

Results: A total of 2074 patients were enrolled. Finally, white blood cell (WBC), C-reactive protein (CRP), interleukin-6 (IL-6), procalcitonin (PCT) and neutrophil-to-lymphocyte ratio (NLR) were included in our models. The AUC of the nomogram model and the simple risk scoring model were 0.854 and 0.842 , respectively. The prediction performance of the two models on sepsis is comparable $(\mathrm{p}=0.1298)$.

Conclusion: This study combining five commonly available inflammatory markers (WBC, CRP, IL-6, PCT and NLR) developed a nomogram model and a simple risk scoring model to predict sepsis in critically ill patients. Although the prediction performance of the two models is comparable, the simple risk scoring model may be simpler and more practical for clinicians to identify potential sepsis in critically ill patients at an early stage and strategize treatments.
\end{abstract}

Keywords: nomogram, score, model, prediction, inflammatory marker, sepsis

\section{Introduction}

Sepsis, associated with a dysregulated host response to infection, is an important global health problem and a major cause of death around the world. ${ }^{1}$ It is estimated that about 48.9 million patients are diagnosed with sepsis, and 11.0 million patients died due to sepsis every year, accounting for approximately $20 \%$ of the global deaths. ${ }^{2,3}$ Although mortality in sepsis remains high, it is treatable. Timely targeted interventions, including antibiotics administration, removal of the source of infection, full fluid resuscitation, and other supportive treatments can reduce mortality and improve prognosis. ${ }^{4-7}$ Therefore, it is important to identify sepsis early and conduct adequate and timely interventions.

Sepsis is induced by infection, involving complex inflammatory responses during its progress, which is accompanied by pro-inflammatory and anti-inflammatory mechanisms. ${ }^{8,9}$ Conventional inflammatory markers, such as procalcitonin (PCT), interleukin-6 (IL-6), white blood cell (WBC), and C-reactive protein (CRP), may be served as potential markers to help clinicians identify sepsis early. ${ }^{10-14}$ Neutrophil-to-lymphocyte ratio (NLR), an easily accessible biomarker that can be calculated from components of the differential white cell count (dividing neutrophil by lymphocyte count), has 
shown good predictive and prognostic performance in various diseases, and some studies have focused on the performance of NLR in the early diagnosis of sepsis. ${ }^{15-19}$ However, to date, none of these markers has gained unanimous independent acceptance for identification of sepsis. Although some previous studies have combined different markers to predict sepsis, the combination of WBC, IL-6, PCT, CRP and NLR to develop predictive models of sepsis in critically ill patients has not been explored.

Nomogram model, which can reduce statistical predictive models into a single numerical estimate of the probability of an event, are widely used to predict diagnosis, staging and prognosis in various situations. ${ }^{20}$ Therefore, we conducted this study to develop and validate a nomogram model and a simple risk scoring model for prediction of sepsis in critically ill patients. Furthermore, we compared the predictive performance of the two models. The aim was to find a model with strong clinical practicability, which can assist clinicians in identifying potential sepsis at an early stage.

\section{Materials and Methods}

\section{Data Source and Patient Selection}

This retrospective study was conducted in the intensive care unit (ICU) of the First Medical Center of the Chinese People's Liberation Army General Hospital, a 3000-bed tertiary teaching hospital in Beijing, China. The medical records of adult critically ill patients admitted to ICU from August 2017 to December 2020 were analyzed. We excluded patients who: 1) Were younger than 18 years old; 2) Were pregnant; 3) Discharged early within 48 hours after ICU admission; 4) Were readmitted to the ICU; 5) Were immunosuppressed; 6) Had hematological malignancy; or 7) Were undergoing cardiopulmonary resuscitation before ICU admission.

For this study, sepsis was diagnosed according to the latest Sepsis-3 definitions. ${ }^{1}$ All sepsis patients received standard-of-care managements in our ICU following the guidelines of 2016 Surviving Sepsis Campaign. ${ }^{21}$ The study complied with the Declaration of Helsinki and has been approved by the Ethical Committee of our hospital (S2017-05402). Considering that this was a retrospective observational study and the personal identifying information was not included, the Institutional Review Committee granted a waiver of informed consent for this study.

\section{Data Collection}

Variables included demographics (age, gender, body mass index (BMI)), vital signs at ICU admission (temperature, systolic blood pressure (SBP), respiratory rate (RR) and heart rate (HR)), Sequential Organ Failure Assessment (SOFA) scores, comorbidities, and the results of the first laboratory test after ICU admission (blood routine tests, blood biochemical tests, arterial blood gas tests, CRP, PCT, IL-6) were collected from hospital electronic medical system.

\section{Laboratory Tests}

Blood samples were obtained on admission to the ICU. NLR and WBC could be obtained from routine blood tests, and the routine blood tests were performed by using an automated hematology analyzer (Sysmex-XN2000, Kobe, Japan). The CRP was examined by using the CRP quantitative analyzer (PA990, Shenzhen, China). The PCT was measured by using the electrochemical luminescence immunoassay system (Roche, cobas 602), and the IL- 6 was measured by the SiemensIMMULT2K.

\section{Statistical Analysis}

For the continuous variables, the Kolmogorov-Smirnov test was performed to test the normal distribution. Continuous variables were expressed as median (interquartile range) or mean (standard deviation), as appropriate, and compared by Mann-Whitney $U$-test and Student $t$-test for non-normally distributed data and for normally distributed data, respectively. Categorical variables were expressed as frequencies (percentages) and the differences between two groups were compared by using chi-square test.

To examine the association between sepsis and the inflammatory markers (WBC, IL-6, PCT, CRP and NLR), both univariate logistic regression analyses and multivariable logistic regression analyses using forward stepwise regression 
were performed with results were expressed as odds ratios (ORs) and 95\% confidence intervals (CIs). A P-value less than or equal to 0.05 was considered statistically significant.

\section{Development and Validation of the Nomogram}

Patients were randomly divided into a training cohort (70\%) and a validation cohort (30\%). Based on the results of the logistic regression, the training cohort was used to construct a nomogram by the "rms" package of $\mathrm{R}$ software. The receiver operating characteristic curves (ROC) were constructed and the area under the curve (AUC) was calculated to evaluate the discrimination performance of the nomogram model. A calibration curve was performed with 1000 bootstrap resampling to compare the predictive probability of the sepsis with the observed incidence of sepsis. The validation cohort was used to assess the generality and stability of the nomogram model. The probability of sepsis was calculated for each patient in the validation cohort according to the established nomogram model. Then, an ROC curve constructed, and finally, the AUC was calculated.

\section{Development and Validation of the Simple Risk Scoring Model}

We also used the training cohort (70\%) and validation cohort (30\%) of the nomogram model to develop and validate a simple risk scoring model for early detection of sepsis. To get a quick score for each variable and finally calculate the total score, all continuous variables included in the model were transformed into dichotomous variables. Considering that either an elevated or a decreased in WBC may indicate infection, it was transformed into a dichotomous variable according to whether its value was within the normal range $\left(4 \sim 10^{*} 10^{9} / \mathrm{L}\right)$. Other continuous variables (IL-6, CRP, PCT, and NLR) were transformed into dichotomous variables based on their optimal cut-off values for the identification of sepsis alone in the training cohort. For the training cohort, a multivariable logistic regression analysis was performed and the OR for each variable was used to construct the simple risk scoring model. ${ }^{22,23}$ Then, the ROC curve was constructed and the AUC was calculated to evaluate the discrimination performance of this scoring model. The optimal cut-off value of the score for predicting sepsis was determined using the Youden index. Finally, this simple risk scoring model was used to calculate risk scores for each patient in the validation cohort, and the AUC was derived based on the risk scores.

The predictive performance between two models and SOFA score were compared by AUC test, which could be achieved by MedCalc (version 19.0.4, Ostend, Belgium). Statistical analyses in this study were performed by SPSS (version 17.0, Chicago, USA), R software (version 4.1.0, Vienna, Austria) and MedCalc (version 19.0.4, Ostend, Belgium).

\section{Results}

\section{Patient Selection and Characteristics}

During the study period, 2514 patients were admitted in ICU. According to our inclusion and exclusion criteria, 2074 patients were included in study. Of these, 1451 patients were randomly assigned to the training cohort and 623 to the validation cohort (Figure 1). The baseline characteristics of the patients in two cohorts were similar, as shown in Table 1. The incidence of sepsis was 688/1451 (47.4\%) in the training cohort and 284/623 (45.6\%) in the validation cohort. No significant difference was detected $(\mathrm{p}=0.444)$.

\section{Results of Logistic Regression Analyses in the Training Cohort}

After univariate logistic and multivariable logistic regression analyses, all of these five markers (WBC, CRP, IL-6, PCT, and NLR) were the independently associated with sepsis. The optimal cut-off values for CRP, IL-6, PCT and NLR identifying sepsis alone were $1.3 \mathrm{mg} / \mathrm{dl}, 90 \mathrm{pg} / \mathrm{mL}, 0.5 \mathrm{ng} / \mathrm{mL}$ and 15 , respectively. Then, they were transformed into dichotomous variables according to the cut-off values. Furthermore, another multivariable logistic regression analysis was conducted based on dichotomous variables. The adjusted ORs for WBC, CRP, IL-6, PCT and NLR were 2.13 (95\% CI: 1.63-2.78), 2.32 (95\% CI: 1.76-3.05), 2.82 (95\% CI: 2.17-3.67), 4.74 (95\% CI: 3.52-6.39) and 4.66 (95\% CI: $3.56-$ 6.10), respectively (Table 2 ). 


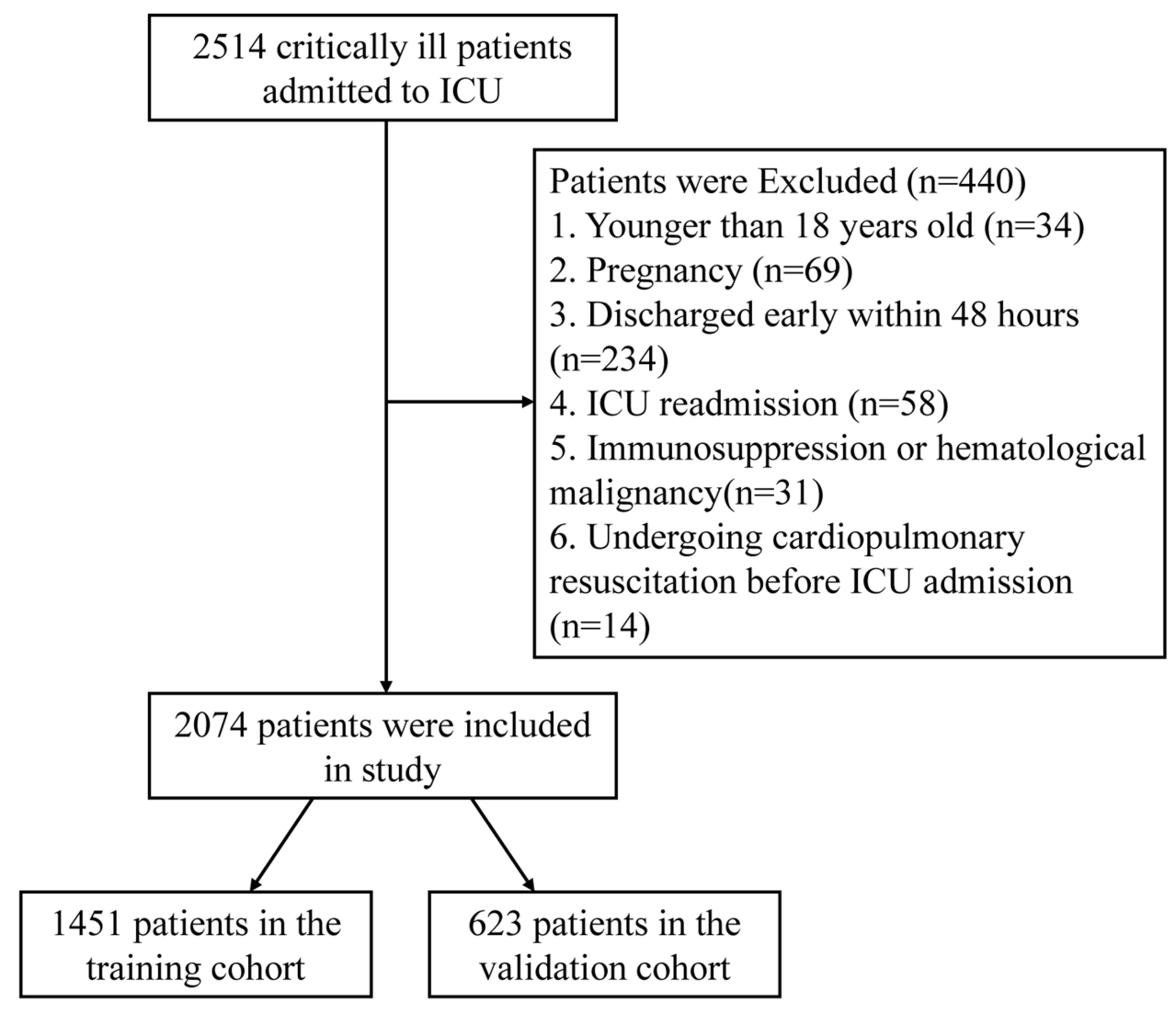

Figure I Flowchart of the enrolled patients. According to our inclusion and exclusion criteria, 2074 patients were included in study. Of these, I45I patients were randomly assigned to the training cohort and 623 to the validation cohort.

\section{Performance of the Nomogram}

According to the results of logistic regression analyses, the first laboratory test results of WBC, CRP, IL-6, PCT and NLR after ICU admission were used to construct nomogram (Figure 2). In the training cohort, the nomogram showed a good discrimination for sepsis, with an AUC 0.854 (95\% CI: 0.835-0.872). At the optimal cut-off value, the sensitivity and specificity were $82.0 \%$ and $73.7 \%$, respectively (Figure $3 \mathrm{~A})$. In the validation cohort, the AUC was 0.857 (95\% CI: $0.827-0.883)$. There was no significant difference between two cohorts in terms of the discrimination $(\mathrm{P}=0.879$; Figure 3A). Furthermore, we compared the discrimination between nomogram and SOFA scores, and nomogram showed a better discrimination $(\mathrm{P}<0.0001$; Figure $3 \mathrm{C})$. The calibration curve presented a good agreement between the nomogram prediction and actual observed for incidence of sepsis (Figure 4).

\section{Performance of the Simple Risk Scoring Model}

Based on the adjusted ORs for WBC, CRP, IL-6, PCT and NLR, we developed a simple risk scoring model, with the total scores ranging from 0 to 16.5 points (Table 2). The model showed good discrimination for sepsis in both the training cohort and the validation cohort, with an AUC 0.842 (95\% CI: 0.822-0.861) and 0.847 (95\% CI: $0.816-$ 0.874). No significant difference was detected between the two cohorts in terms of the discrimination $(\mathrm{p}=0.7941$; Figure 3B). In the training cohort, the optimal cut-off value was 7.5 points, with a sensitivity $77.03 \%$ and specificity $75.75 \%$. Then, we compared the simple risk scoring model with nomogram and SOFA scores for sepsis discrimination. Results indicated the simple risk scoring model was comparable with the nomogram in the discrimination of sepsis $(\mathrm{p}=0.1298$; Figure 3C), and the simple risk scoring model performed better than SOFA scores in the discrimination of sepsis $(\mathrm{p}<0.0001$; Figure $3 \mathrm{C})$. 
Table I Baseline Characteristics of the Training Cohort and Validation Cohort

\begin{tabular}{|c|c|c|c|}
\hline Variable & Training Cohort $(n=|45|)$ & Validation Cohort $(n=623)$ & $P$ value \\
\hline \multicolumn{4}{|l|}{ Demographics } \\
\hline Age, years (IQR) & $64(52,74)$ & $64(51,74)$ & 0.562 \\
\hline Male $(\%)$ & $892(61.5)$ & $390(62.6)$ & 0.629 \\
\hline $\mathrm{BMI}, \mathrm{Kg} / \mathrm{m}^{2}$ (IQR) & $23.56(21.16,26.03)$ & $23.53(21.22,25.95)$ & 0.792 \\
\hline \multicolumn{4}{|l|}{ Vital signs (IQR) } \\
\hline Temperature, ${ }^{\circ} \mathrm{C}$ & $36.2(36.0,36.5)$ & $36.2(36.0,36.5)$ & 0.792 \\
\hline $\mathrm{SBP}, \mathrm{mmHg}$ & $|3|(|13| 49)$, & $13 \mid(112,149)$ & 0.628 \\
\hline $\mathrm{HR}$, bpm & $89(75,102)$ & $88(76,102)$ & 0.669 \\
\hline $\mathrm{RR}, \mathrm{bpm}$ & $16(15,19)$ & $16(15,19)$ & 0.535 \\
\hline Sepsis (\%) & $688(47.4)$ & $284(45.6)$ & 0.444 \\
\hline SOFA (IQR) & $4(2,7)$ & $4(2,6)$ & 0.226 \\
\hline \multicolumn{4}{|l|}{ Comorbidities (\%) } \\
\hline Hypertension & $586(40.4)$ & $247(39.6)$ & 0.753 \\
\hline Diabetes & $290(20.0)$ & $125(20.1)$ & 0.968 \\
\hline CHD & $245(16.9)$ & $108(17.3)$ & 0.802 \\
\hline $\mathrm{CHF}$ & $49(3.4)$ & $22(3.5)$ & 0.859 \\
\hline Cerebral vascular disease & $146(10.1)$ & $79(12.7)$ & 0.079 \\
\hline Chronic pulmonary disease & $74(5.1)$ & $31(5.0)$ & 0.906 \\
\hline Liver disease & $81(5.6)$ & $40(6.4)$ & 0.455 \\
\hline Renal disease & $87(6.0)$ & $48(7.7)$ & 0.148 \\
\hline \multicolumn{4}{|l|}{ First laboratory tests (IQR) } \\
\hline WBC $\left(* 10^{9} / \mathrm{L}\right)$ & $10.34(7.49,13.93)$ & $10.56(7.98,13.88)$ & 0.400 \\
\hline IL-6 (pg/mL) & $90.26(36.96,238.50)$ & $78.35(33.22,212.80)$ & 0.079 \\
\hline $\mathrm{Hb}(\mathrm{g} / \mathrm{L})$ & $104(89,119)$ & $103(88,121)$ & 0.859 \\
\hline $\operatorname{PLT}\left(* 10^{9} / \mathrm{L}\right)$ & $172(|25,23|)$ & $184(133,252)$ & 0.001 \\
\hline NLR & $|3.2|(7.88,22.12)$ & $12.96(7.69,21.65)$ & 0.866 \\
\hline CRP (mg/dl) & $1.55(0.27,5.01)$ & $1.30(0.22,5.08)$ & 0.480 \\
\hline $\mathrm{BNP}(\mathrm{pg} / \mathrm{mL})$ & $242.20(87.20,918.00)$ & $235.50(87.20,814.00)$ & 0.544 \\
\hline $\mathrm{ALT}(\mathrm{u} / \mathrm{L})$ & $18.80(11.00,38.80)$ & $19.30(11.00,39.50)$ & 0.924 \\
\hline AST $(u / L)$ & $24.50(16.00,47.70)$ & $24.20(16.10,47.20)$ & 0.988 \\
\hline Alb $(g / L)$ & $30.90(27.00,34.50)$ & $31.20(27.70,35.00)$ & 0.086 \\
\hline DBil $(\mu \mathrm{mol} / \mathrm{L})$ & $5.90(3.60,10.10)$ & $5.60(3.50,8.90)$ & 0.064 \\
\hline TBil $(\mu \mathrm{mol} / \mathrm{L})$ & $13.00(8.30,19.80)$ & $12.40(8.40,19.00)$ & 0.297 \\
\hline $\mathrm{SCr}(\mu \mathrm{mol} / \mathrm{L})$ & $70.40(54.50,93.70)$ & $70.30(55.50,92.10)$ & 0.844 \\
\hline PCT (ng/mL) & $0.20(0.06,0.97)$ & $0.15(0.06,0.82)$ & 0.080 \\
\hline Lac (mmol/L) & $1.60(1.10,2.70)$ & $1.60(1.00,2.50)$ & 0.168 \\
\hline
\end{tabular}

Abbreviations: IQR, interquartile range; BMI, body mass index; SBP, systolic blood pressure; HR, heart rate; RR, respiratory rate; CHD, coronary heart disease; SOFA, Sequential Organ Failure Assessment; CHF, chronic heart failure; WBC, white blood cell; IL-6, interleukin-6; Hb, hemoglobin; PLT, platelet; NLR, neutrophil-tolymphocyte ratio; CRP, C-reactive protein; BNP, B-type natriuretic peptide; ALT, alanine aminotransferase; AST, aspartate aminotransferase; Alb, albumin; DBil, direct bilirubin; TBil, total bilirubin; SCr, serum creatinine; PCT, procalcitonin; Lac, lactic acid.

\section{Discussion}

Sepsis is a complex disorder with high morbidity and mortality, and remains a global health priority. ${ }^{24}$ Early identification of sepsis can facilitate timely clinical interventions and may improve prognosis. ${ }^{25}$ Our study indicated that inflammatory markers, including WBC, CRP, IL-6, PCT and NLR, were the independently associated with sepsis in critically ill patients admitted to ICU. Furthermore, a nomogram and a simple risk scoring model were constructed. The prediction performance of the two models on sepsis was comparable to each other and provided better prediction than that of SOFA score.

To our knowledge, early detection of sepsis is necessary, so that specific goal-directed therapy bundles to minimize complications may be initiated; hence, many studies have focused on early sepsis detection. Some have used biomarkers, some have built scoring models, and in recent years, an increasing number of studies have focused on machine 

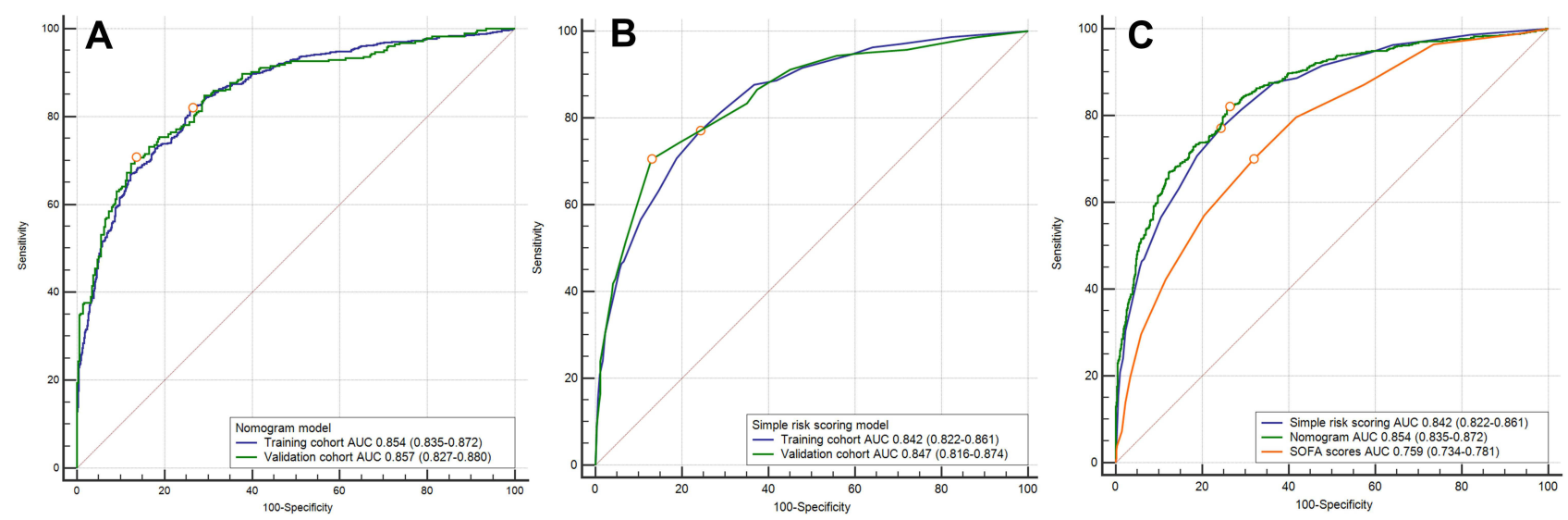

Figure 3 Receiver operating characteristic curve analyses of models for predicting sepsis. (A) Nomogram model; (B) the simple risk scoring model; (C) Nomogram model, simple risk scoring model and SOFA scores.
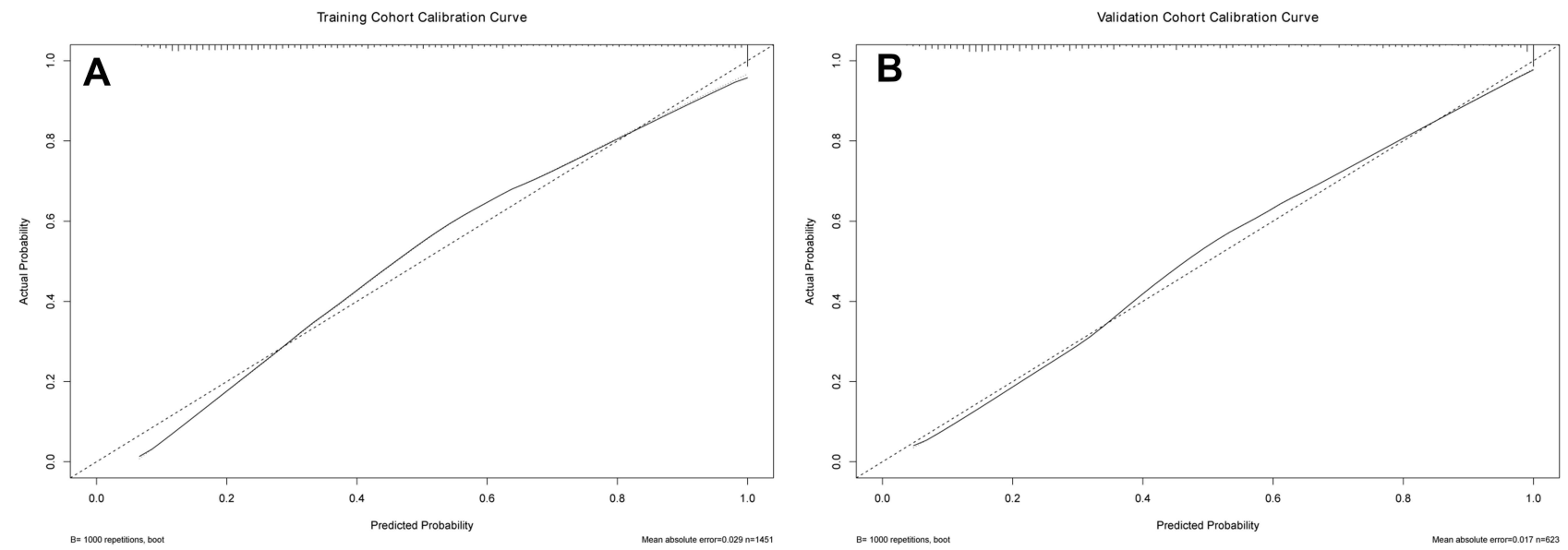

Figure 4 Calibration curves for nomogram model in the training cohort $(\mathbf{A})$ and validation cohort $(\mathbf{B})$. In the calibration curve, the $\mathrm{X}$-axis represents the predicted probability of sepsis, and the Y-axis represents the actual sepsis incidence rate. The $45^{\circ}$ diagonal dotted line represents ideal predictions. The solid line represents the performance of the nomogram model, of which a closer fit to the diagonal dotted line represents a better prediction.

Sepsis is a systemic inflammatory response due to infection, resulting in organ dysfunction. Inflammation plays a crucial role in the process of sepsis. Our study combined inflammatory markers, including WBC, CRP, IL-6, PCT and NLR, to predict sepsis. WBC is the most common inflammatory marker in clinic, and WBC count greater than 12,000 or less than 4000 /microliters is one of the criteria for the diagnosis of systemic inflammatory response syndrome (SIRS) ${ }^{28}$ It is well established that CRP is an acute inflammatory marker that acts as part of the acute-phase reaction in sepsis, and its concentration increases during infection. ${ }^{29,30} \mathrm{CRP}$ has a half-life of 19 hours. It is produced within 4-6 hours after inflammation onset and peaks at 36-48 hours. ${ }^{31,32}$ IL-6 is an important proinflammatory cytokine produced early in inflammation and plays a role in the complex pathophysiology process of sepsis. ${ }^{33,34}$ IL-6 levels begin to rise within 1 hour of the infectious stimulus and peaked at 2 hours. ${ }^{35}$ PCT has attracted much attention due to its high accuracy in sepsis diagnosis and prognosis. ${ }^{36}$ PCT is secreted by many cell types during systemic inflammation of infectious origin, and the level is generally low in nonbacterial origin systemic inflammation. ${ }^{37}$ The half-life of PCT is 24 hours. Its level begins to rise within 2-4 hours after systemic inflammation and peaks at 12-48 hours, making it valuable in the early detection of sepsis. ${ }^{38}$ In our study, the weight of PCT is the greatest among five inflammatory markers in both nomogram model and the simple risk scoring model. In the simple risk scoring model, it would be assigned 4.5 points when greater than the cut-off value. During the progress of sepsis, apoptosis-induced lymphopenia is a prominent feature of sepsis, and neutrophils are recruited to control infection. ${ }^{39-42}$ A dramatical increase in NLR can be observed in sepsis. These 
markers are readily available and routinely measured at admission to our ICU. However, using any of above-mentioned markers alone to identify sepsis may lack sensitivity and specificity. Therefore, we developed and validated a nomogram model including all the above markers to predict sepsis in critically ill patients.

Although some previous studies have constructed nomogram models in predicting sepsis, there has been no study combining the results of WBC, CRP, IL-6, PCT and NLR measured at ICU admission to predict sepsis. ${ }^{43-46}$ To our knowledge, these five inflammatory markers are routinely monitored in the ICU for patients with suspected infection and the results can be obtained quickly. Our nomogram model showed good prediction performance as indicated by the AUC value, which is better than that of SOFA score $(\mathrm{P}<0.0001)$. In addition, our nomogram model has fewer items and is more convenient to use compared with the SOFA score. The calibration curves presented a good agreement between the nomogram prediction and actual observed for incidence of sepsis. Nomogram is a visualized, reliable and intuitive graphical tool for predicting and quantifying risk of individual experiencing a clinical event based on relevant factors; however, sometimes bedside applications are not so convenient. Therefore, we developed a simple risk scoring model based on dichotomous variables and its prediction performance was comparable to that of the nomogram, which can somewhat help clinicians quickly determine the risk of sepsis at an early stage.

This is the first study to construct a nomograph predictive model of sepsis in ICU using five commonly available inflammatory markers. Furthermore, a simple risk scoring model with good prediction performance is constructed. This study also has some limitations. First, this is a single-center retrospective study that still requires external validation. The model needs other centers to further evaluate. Secondly, the study mainly focused on ICU patients, which may not be suitable for emergency patients. Thirdly, to make the model simple and practical, we did not include other laboratory tests other than inflammatory markers, which may potentially have reduced the performance of the model.

\section{Conclusion}

This study used five commonly available inflammatory markers, including WBC, CRP, IL-6, PCT and NLR, to develop a nomogram model and a simple risk scoring model to predict sepsis in critically ill patients. Although the prediction performance of the two models is comparable, the simple risk scoring model may be more practical for clinicians to identify potential sepsis in critically ill patients at an early stage and strategize treatments.

\section{Abbreviations}

AUC, area under the curve; BMI, body mass index; CRP, C-reactive protein; CIs, confidence intervals; HR, heart rate; ICU, intensive care unit; IL-6, interleukin-6; NLR, neutrophil-to-lymphocyte ratio; OR, odds ratio; PCT, procalcitonin; RR, respiratory rate; ROC, receiver operating characteristic curves; SOFA, Sequential Organ Failure Assessment; SBP, systolic blood pressure; SIRS, systemic inflammatory response syndrome; WBC, white blood cell.

\section{Data Sharing Statement}

The cohorts generated and/or analyzed during this study are not publicly available, due to currently ongoing research studies, but the data are available from the corresponding author on reasonable request.

\section{Ethics Approval and Consent to Participate}

This study complied with the Declaration of Helsinki and has been approved by the Ethical Committee of the First Medical Centre of the Chinese PLA General Hospital, Beijing, China (S2017-054-02). Considering this was a retrospective observational study and the personal identifying information was not included, the Institutional Review Committee of our hospital granted a waiver of informed consent for this study. The patient data was maintained with confidentiality.

\section{Acknowledgments}

The authors would like to thank all the doctors, nurses, technicians, and patients involved at the participating center for their dedication in the study. 


\section{Author Contributions}

All authors made substantial contributions to conception and design, acquisition of data, or analysis and interpretation of data; took part in drafting the article or revising it critically for important intellectual content; agreed to submit to the current journal; gave final approval of the version to be published; and agree to be accountable for all aspects of the work.

\section{Funding}

Innovation Research of Chinese PLA general hospital (CX19010), Special Research of Military Medical Innovation (18CXZ026), and Special Research Fund for Health Care (20BJZ27).

\section{Disclosure}

The authors declare that they have no competing interests.

\section{References}

1. Singer M, Deutschman CS, Seymour CW, et al. The third international consensus definitions for sepsis and septic shock (sepsis-3). JAMA. 2016;315(8):801-810. doi:10.1001/jama.2016.0287

2. Rudd KE, Johnson SC, Agesa KM, et al. Global, regional, and national sepsis incidence and mortality, 1990-2017: analysis for the Global Burden of Disease Study. Lancet. 2020;395(10219):200-211. doi:10.1016/S0140-6736(19)32989-7

3. Fleischmann-Struzek C, Schwarzkopf D, Reinhart K. Sepsis incidence in Germany and worldwide: current knowledge and limitations of research using health claims data. Med Klin Intensivmed Notfmed. 2021;1-5. doi:10.1007/s00063-021-00777-5

4. Seymour CW, Gesten F, Prescott HC, et al. Time to treatment and mortality during mandated emergency care for sepsis. $N$ Engl J Med. 2017;376 (23):2235-2244. doi:10.1056/NEJMoa1703058

5. Prasad PA, Shea ER, Shiboski S, Sullivan MC, Gonzales R, Shimabukuro D. Relationship between a sepsis intervention bundle and in-hospital mortality among hospitalized patients: a retrospective analysis of real-world data. Anesth Analg. 2017;125(2):507-513. doi:10.1213/ ANE.0000000000002085

6. Liu VX, Fielding-Singh V, Greene JD, et al. The timing of early antibiotics and hospital mortality in sepsis. Am J Respir Crit Care Med. 2017;196 (7):856-863. doi:10.1164/rccm.201609-18480C

7. Kahn JM, Davis BS, Yabes JG, et al. Association between state-mandated protocolized sepsis care and in-hospital mortality among adults with sepsis. JAMA. 2019;322(3):240-250. doi:10.1001/jama.2019.9021

8. Venet F, Monneret G. Advances in the understanding and treatment of sepsis-induced immunosuppression. Nat Rev Nephrol. 2018;14(2):121-137. doi:10.1038/nrneph.2017.165

9. Lelubre C, Vincent JL. Mechanisms and treatment of organ failure in sepsis. Nat Rev Nephrol. 2018;14(7):417-427. doi:10.1038/s41581-018-0005-7

10. Wacker C, Prkno A, Brunkhorst FM, Schlattmann P. Procalcitonin as a diagnostic marker for sepsis: a systematic review and meta-analysis. Lancet Infect Dis. 2013;13(5):426-435. doi:10.1016/S1473-3099(12)70323-7

11. Harbarth S, Holeckova K, Froidevaux C, et al. Diagnostic value of procalcitonin, interleukin-6, and interleukin-8 in critically ill patients admitted with suspected sepsis. Am J Respir Crit Care Med. 2001;164(3):396-402. doi:10.1164/ajrccm.164.3.2009052

12. Tessema B, Lippmann N, Willenberg A, Knupfer M, Sack U, Konig B. The diagnostic performance of interleukin-6 and C-reactive protein for early identification of neonatal sepsis. Diagnostics. 2020;10(11). doi:10.3390/diagnostics10110978

13. Stocker M, van Herk W, El Helou S, et al. C-reactive protein, procalcitonin, and white blood count to rule out neonatal early-onset sepsis within 36 hours: a secondary analysis of the neonatal procalcitonin intervention study. Clin Infect Dis. 2020. doi:10.1093/cid/ciaa290

14. Molano Franco D, Arevalo-Rodriguez I, Roque IFM, Montero Oleas NG, Nuvials X, Zamora J. Plasma interleukin-6 concentration for the diagnosis of sepsis in critically ill adults. Cochrane Database Syst Rev. 2019;4:CD011811. doi:10.1002/14651858.CD011811.pub2

15. Huang Z, Fu Z, Huang W, Huang K. Prognostic value of neutrophil-to-lymphocyte ratio in sepsis: a meta-analysis. Am J Emerg Med. 2020;38 (3):641-647. doi:10.1016/j.ajem.2019.10.023

16. Li X, Liu C, Mao Z, et al. Predictive values of neutrophil-to-lymphocyte ratio on disease severity and mortality in COVID-19 patients: a systematic review and meta-analysis. Crit Care. 2020;24(1):647. doi:10.1186/s13054-020-03374-8

17. Kim S, Eliot M, Koestler DC, Wu WC, Kelsey KT. Association of neutrophil-to-lymphocyte ratio with mortality and cardiovascular disease in the Jackson Heart Study and modification by the Duffy antigen variant. JAMA Cardiol. 2018;3(6):455-462. doi:10.1001/jamacardio.2018.1042

18. Sumitro KR, Utomo MT, Widodo ADW. Neutrophil-to-lymphocyte ratio as an alternative marker of neonatal sepsis in developing countries. Oman Med J. 2021;36(1):e214. doi:10.5001/omj.2021.05

19. Westerdijk K, Simons KS, Zegers M, Wever PC, Pickkers P, de Jager CPC. The value of the neutrophil-lymphocyte count ratio in the diagnosis of sepsis in patients admitted to the intensive care unit: a retrospective cohort study. PLoS One. 2019;14(2):e0212861. doi:10.1371/journal. pone. 0212861

20. Iasonos A, Schrag D, Raj GV, Panageas KS. How to build and interpret a nomogram for cancer prognosis. J Clin Oncol. 2008;26(8):1364-1370. doi:10.1200/JCO.2007.12.9791

21. Rhodes A, Evans LE, Alhazzani W, et al. Surviving sepsis campaign: international guidelines for management of sepsis and septic shock: 2016. Intensive Care Med. 2017;43(3):304-377. doi:10.1007/s00134-017-4683-6

22. Tsui TL, Huang YT, Kan WC, et al. A novel procalcitonin-based score for detecting sepsis among critically ill patients. PLoS One. 2021;16(1): e0245748. doi:10.1371/journal.pone.0245748 
23. Zhang K, Zhang S, Cui W, Hong Y, Zhang G, Zhang Z. Development and validation of a sepsis mortality risk score for sepsis-3 patients in intensive care unit. Front Med. 2020;7:609769. doi:10.3389/fmed.2020.609769

24. Reinhart K, Daniels R, Kissoon N, Machado FR, Schachter RD, Finfer S. Recognizing sepsis as a global health priority - a WHO resolution. $N$ Engl J Med. 2017;377(5):414-417. doi:10.1056/NEJMp1707170

25. Cecconi M, Evans L, Levy M, Rhodes A. Sepsis and septic shock. Lancet. 2018;392(10141):75-87. doi:10.1016/S0140-6736(18)30696-2

26. Nesaragi N, Patidar S. Early prediction of sepsis from clinical data using ratio and power-based features. Crit Care Med. $2020 ; 48(12)$ :e1343-e1349. doi:10.1097/CCM.0000000000004691

27. Fleuren LM, Klausch TLT, Zwager CL, et al. Machine learning for the prediction of sepsis: a systematic review and meta-analysis of diagnostic test accuracy. Intensive Care Med. 2020;46(3):383-400. doi:10.1007/s00134-019-05872-y

28. Chakraborty RK, Burns B. Systemic Inflammatory Response Syndrome. Treasure Island, FL: StatPearls; 2021.

29. Healy B, Freedman A. Infections. BMJ. 2006;332(7545):838-841. doi:10.1136/bmj.332.7545.838

30. Sproston NR, Ashworth JJ. Role of C-reactive protein at sites of inflammation and infection. Front Immunol. 2018;9:754. doi:10.3389/ fimmu. 2018.00754

31. Prucha M, Bellingan G, Zazula R. Sepsis biomarkers. Clin Chim Acta. 2015;440:97-103. doi:10.1016/j.cca.2014.11.012

32. Eschborn S, Weitkamp JH. Procalcitonin versus C-reactive protein: review of kinetics and performance for diagnosis of neonatal sepsis. J Perinatol. 2019;39(7):893-903. doi:10.1038/s41372-019-0363-4

33. Schulte W, Bernhagen J, Bucala R. Cytokines in sepsis: potent immunoregulators and potential therapeutic targets-an updated view. Mediators Inflamm. 2013;2013:165974. doi:10.1155/2013/165974

34. Kishimoto T. IL-6: from its discovery to clinical applications. Int Immunol. 2010;22(5):347-352. doi:10.1093/intimm/dxq030

35. Ma L, Zhang H, Yin YL, et al. Role of interleukin-6 to differentiate sepsis from non-infectious systemic inflammatory response syndrome. Cytokine. 2016;88:126-135. doi:10.1016/j.cyto.2016.08.033

36. Gregoriano C, Heilmann E, Molitor A, Schuetz P. Role of procalcitonin use in the management of sepsis. J Thorac Dis. 2020;12(Suppl 1):S5-S15. doi: $10.21037 /$ jtd.2019.11.63

37. Meisner M. Pathobiochemistry and clinical use of procalcitonin. Clin Chim Acta. 2002;323(1-2):17-29. doi:10.1016/S0009-8981(02)00101-8

38. Malik M, Sreekantan Nair A, Illango J, et al. The advancement in detecting sepsis and its outcome: usefulness of procalcitonin in diagnosing sepsis and predicting fatal outcomes in patients admitted to intensive care unit. Cureus. 2021;13(4):e14439. doi:10.7759/cureus.14439

39. Girardot T, Rimmele T, Venet F, Monneret G. Apoptosis-induced lymphopenia in sepsis and other severe injuries. Apoptosis. 2017;22(2):295-305. doi:10.1007/s10495-016-1325-3

40. Santos I, Colaco HG, Neves-Costa A, et al. CXCL5-mediated recruitment of neutrophils into the peritoneal cavity of Gdf15-deficient mice protects against abdominal sepsis. Proc Natl Acad Sci U S A. 2020;117(22):12281-12287. doi:10.1073/pnas.1918508117

41. de Pablo R, Monserrat J, Prieto A, Alvarez-Mon M. Role of circulating lymphocytes in patients with sepsis. Biomed Res Int. $2014 ; 2014: 671087$. doi: $10.1155 / 2014 / 671087$

42. Shen XF, Cao K, Jiang JP, Guan WX, Du JF. Neutrophil dysregulation during sepsis: an overview and update. J Cell Mol Med. 2017;21 (9):1687-1697. doi:10.1111/jcmm.13112

43. Zhang L, Zhang F, Xu F, et al. Construction and evaluation of a sepsis risk prediction model for urinary tract infection. Front Med. 2021 ;8:671184. doi:10.3389/fmed.2021.671184

44. Wang J, Mi Y, Wu S, Shao H, Zhu L, Dai F. Impact factors and an efficient nomogram for predicting the occurrence of sepsis after percutaneous nephrolithotomy. Biomed Res Int. 2020;2020:6081768. doi:10.1155/2020/6081768

45. Zhang H, Meng F, Lu S. Nomograms predicting the occurrence of sepsis in patients following major hepatobiliary and pancreatic surgery. Gastroenterol Res Pract. 2020;2020:9761878. doi:10.1155/2020/9761878

46. Mearelli F, Fiotti N, Giansante C, et al. Derivation and validation of a biomarker-based clinical algorithm to rule out sepsis from noninfectious systemic inflammatory response syndrome at emergency department admission: a multicenter prospective study. Crit Care Med. 2018;46 (9):1421-1429. doi:10.1097/CCM.0000000000003206

\section{Publish your work in this journal}

The International Journal of General Medicine is an international, peer-reviewed open-access journal that focuses on general and internal medicine, pathogenesis, epidemiology, diagnosis, monitoring and treatment protocols. The journal is characterized by the rapid reporting of reviews, original research and clinical studies across all disease areas. The manuscript management system is completely online and includes a very quick and fair peer-review system, which is all easy to use. Visit http://www.dovepress.com/testimonials.php to read real quotes from published authors.

Submit your manuscript here: https://www.dovepress.com/international-journal-of-general-medicine-journal 